\title{
ON THE EXISTENCE OF NON-COMPARABLE HOMOGENEOUS TOPOLOGIES WITH THE SAME CLASS OF HOMEOMORPHISMS
}

\author{
YU-LEE LEE
}

(Received September 17, 1969; revised December 8, 1969)

Let $H(X, \mathcal{U})$ be the class of all homeomorphisms from $(X, \mathcal{U})$ onto itself. We have already known that, in general, there exist many topologies $C V$ on $X$ such that $H(X, \mathcal{U})=H(X, \mathcal{Q})[1][2][3]$. If $(X, \mathcal{U})$ is an $n$-manifold, then $H(X, U)$ can also determine, to a certain degree, the topologies $C V$ on $X$ with $H(X, \mathcal{U})=H(X, \mathcal{V})[4]$. However it was unknown that whether there are topologies $\mathcal{U}$ and $\mathcal{V}$ on a set $X$ such that $H(X, \mathcal{U})=H(X, C V),(X, \mathcal{U})$ and $(X, C)$ are homogeneous spaces and $\mathcal{U}$ and $\mathcal{V}$ are non-comparable in the sense that there does not exist a permutation $\Phi$ on $X$ such that $\{\Phi(U) \mid U \in \mathcal{U}\} \cong \mathcal{C V}$ or $\{\Phi(V) \mid V \in \mathcal{V}\}$ $\subseteq \mathcal{U}$. A negative answer would characterize a homogeneous space by means of the class of homeomorphism. The finding of this paper proves the existence of non-comparable homogeneous topological spaces with the same class of homeomorphisms. However it is still open whether there exist non-comparable compact homogeneous topologies with the same class of homeomorphisms.

THEOREM 1. Let $(X, \mathcal{U})$ be an n-manifold and let $C V=\{U \in \mathcal{U} \mid X \backslash U$ is compact\} and let $\mathscr{W}$ be the topology having

$$
\left\{V \backslash \bigcup_{i=1}^{\infty}\left\{p_{i}\right\} \mid V \in \mathcal{Q} \text { and }\left\{p_{i}\right\} \text { converges to } p_{0} \text { in }(X, U)\right\}
$$

as a subbase. Then $H(X, U)=H(X, \mathscr{W})$. If $(X, \mathcal{U})$ is not compact, then $(X, \mathcal{U})$ and $(X, \mathscr{W})$ are non-comparable.

Theorem 1 is a special case of a more general theorem. Let $(X, \mathcal{U})$ be a topological space, a topology $C$ on $X$ is said to be a $C$-topology relative to $U$ if

(i) $H(X, \mathcal{U} \subseteq H(X, \mathcal{Q})$,

(ii) $U \in \mathcal{Q}$ if, and only if $U \cup V \in \mathcal{V}$ for every non-empty $V$ in $\mathcal{C}$.

We have the following lemma. [1] 
LeMmA 1. If $\mathcal{Q}$ is a $C$-topology relative to $\mathcal{U}$ on $X$, then $\mathcal{V} \leqq \mathcal{U}$ and $H(X, \mathcal{Q})=H(X, \mathcal{V})$.

We recall that [3] a family of subsets $\mathscr{N}$ in $(X, \mathcal{U})$ is called an $\Im$-family if the following three conditions are satisfied:

(1) The empty set $\emptyset$ is in $\mathscr{\eta}$.

(2) If $N \in \mathscr{l}$ then Int $(N)=\emptyset$.

(3) If $\left\{N_{1}, \cdots, N_{k}\right\} \subset \Re$ and $\left\{f_{1}, \cdots, f_{k}\right\} \subset H(X, \mathcal{U})$, then $\bigcup\left\{f_{i}\left(N_{i}\right): i=1\right.$, $2, \cdots, k\} \in \mathscr{N}$ for every $k$.

We also have the following result.

LEMMA 2. [3] If $\boldsymbol{\eta}$ is an $\Im$-family of subsets of a regular ' $T$ '-space $(X, \mathcal{Q})$, then $\{U-N \mid U \in \mathcal{U}, N \in \Re\}$ forms a base of a topology $\mathcal{V}$ on $X, \mathcal{U} \subseteq \mathcal{V}$ and $H(X, \mathcal{U})=H(X, \subset)$.

THEOREM 2. Let $\mathcal{Q}$ be a C-topology on a regular $T_{1}$-space $(X, \mathcal{U})$ such that each point $p$ in $X$ has a closed neighborhood $C_{p} \neq X$ with $X \backslash C_{p}$ in $\mathcal{Q}$. Let $\mathscr{W}$ be the topology having $\{V \backslash N \mid V \in C, N \in \mathscr{M}\}$ as a base where $\Re$ is an $\Im$-family of subsets in $(X, \mathcal{U})$ such that $N \in \Re$ and $A \subset N$ implies $A \in \Re$. Then

$$
H(X, \mathcal{U})=H(X, \propto)=H(X, \mathscr{W}) .
$$

ProOF. Let $\mathcal{S}$ be the topology having $\{U \backslash N \mid U \in \mathcal{U}, N \in \mathscr{M}\}$ as a base. We want to show that $\mathscr{W}$ is a $C$-topology in $(X, \mathcal{S})$. If $f \in H(X, \mathcal{S})$,

$$
V \in \mathcal{Q} \text { and } N \in \mathcal{M} \text {, then } f(V \backslash N)=f(V) \backslash f(N) \text {. }
$$

By Lemma 2 and the definition of an $\Im$-family, $f \in H(X, \mathcal{U})$ and $f(N) \in \Re$. By Lemma $1 f \in H(X, C)$ and $f(V) \in \mathcal{U}$. Therefore $f(V \backslash N) \in \mathscr{W}$ and $f$ is an open map in $(X, \mathscr{W})$. Similarly $f$ is continuous in $(X, \mathscr{W})$ and $f \in H(X, \mathscr{W})$. Hence $H(X, \mathcal{S}) \cong H(X, \mathscr{W})$.

If $S \in \mathcal{S}$ and $W \in \mathscr{W}$, then $W=\bigcup_{\alpha}\left\{V_{\alpha} \backslash N_{\alpha}\right\}$ and $S \cup \bigcup_{\alpha}\left\{V_{\alpha} \backslash N_{\alpha}\right\}=$ $\bigcup_{\alpha}\left\{\left(S \cup V_{\alpha}\right) \backslash\left(N_{\alpha} \backslash S\right)\right\} \in \mathscr{W}$.

If $S \notin \mathcal{S}$, then $S$ contains a boundary point $p$ relative to $(X, \mathcal{S})$. By assumption, there exists a closed neighborhood $A \neq X$ of $p$ in $(X, \mathcal{U})$ with $X \backslash A \in C V$. Then $(X \backslash A) \cap S \notin \mathscr{W}$. Since otherwise there exists a neighborhood $V \backslash N$ of $p$ in $\mathscr{W}$ with $V \in \mathcal{C}, N \in \mathscr{N}$ and $p \in V \backslash N \subset(X \backslash A) \cup S$, which implies $p \in(V \cap A) \backslash N \subset S$ and $p$ 
is an interior point of $S$ relative to $(X, \mathcal{S})$.

Hence $\mathscr{W}$ is a $C$-topology in $(X, \mathcal{S})$ and $H(X, \mathcal{S})=H(X, \mathscr{W})=H(X, \mathcal{U})$.

The first half of Theorem 1 is a special case of Theorem2. To prove the non-comparability of $U$ and $\mathscr{W}$, we see that $(X, \mathscr{W})$ is not Hausdorff, hence $\{\Phi(U) \mid U \in \mathcal{U}\} \stackrel{\Phi}{\ddagger} \mathcal{W}$ for any permutation $\Phi$, also $(X, \mathscr{W})$ is not locally compact, hence $\{\Phi(W) \mid W \in \mathscr{W}\} \stackrel{+}{\neq} \mathcal{U}$ for any permutation $\Phi$.

CONJECTURE. In the category of compact homogeneous Hausdorff spaces, the classes of all homeomorphisms characterize the topology.

REMARK. J. V. Whittaker has proved that in the category of compact manifolds, the groups of homeomorphisms characterize the topology.

\section{BIBLIOGRAPHY}

[1] Y. L. LEE, Topologies with the same class of homeomorphisms, Pacific J. Math., 20(1967), 77-83.

[2] Y. L. LEE, Continua with the same class of homeomorphisms, Kyungpook Math. J. 7(1967), $1-4$.

[3] Y.L. LEE, On a class of finer topologies with the same class of homeomorphisms, Proc. Amer. Math. Soc., 21(1969), 129-133.

[4] Y.L. LEE, Characterizing the topology by the class of homeomorphisms, Duke Math. J., 35(1968), 625-630.

[5] J. WHITTAKER, On isomorphic groups and homeomorphic spaces, Ann. of Math., 78(1963), 74-91.

DEPARTMENT OF MATHEMATICS

KANSAS STATE UNIVERSITY

KANSAS, U.S.A.

This research was patially supported by Kansas State University Faculty Summer Research Fellowship Program. 